\title{
Electrocatalytic effects of Pt-based nanoparticles studied with advanced identical location electron microscopy
}

Francisco Ruiz-Zepeda, Armin Havlišič, Andraž Pavlišič, Ana Rebeka Kamšek, Matija Gatalo, Marjan Bele, Primož Jovanovič, Goran Dražić and Nejc Hodnik

National Institute of Chemistry, Ljubljana, Slovenia

The electrocatalytic performance of nanoparticles is usually governed by the surface morphology which is also inherently related to the nanostructure and chemical composition. Therefore, surface and structural analysis during electrochemical processes are necessary to further understand and improve their electrocatalytic properties. Activity and stability in nanoparticles employed in current fuel cells are still considered below the desirable standards and need to be improved further to reach a widely use in the market of electric vehicles [1]. Of particular interest is the behavior and evolution of the electrocatalysts when submitted to an electrochemical process such as an activation protocol [2]. In addition, Pt-based metallic electrocatalysts typically suffer from dissolution or dealloying due to the harsh conditions occurring in a regular workload.

In this work, we present a study of different metallic electrocatalysts examined by identical location (IL) aberration corrected scanning transmission electron microscopy (AC-STEM). This method consists in examining the same area of the sample before and after an electrochemical process has taken place $[2,3]$. Among other phenomena occurring during the electrochemical degradation, highly valuable is witnessing individual nanoparticles that have experienced dissolution or redeposition on certain facets [4, 5], plus retrieving precise information from the distribution of strain in the lattice. By being able to track these changes ex-situ it is possible to gain a better understanding of the different events involved during the electrochemical cycling and provide a better overview of the electrocatalyst stability and activity. Hence the IL-(S)TEM strategy allowed us to observe important structural features that usually are hard to follow if the same nanoparticle is not monitored during the electrochemical processes. Additionally we will discuss the advantages and disadvantages as an alternative method against the in-situ technique [6,7].

\section{References}

[1] K. Kodama, T. Nagai, A. Kuwaki, R. Jinnouchi, Y. Morimoto, Nat. Nanotech. 16 (2021) 140-147.

[2] A. Hrnjić, F. Ruiz-Zepeda, M. Gaberšček, M. Bele, L. Suhadolnik, N. Hodnik, P. Jovanovič, J. Electrochem. Soc. 167 (2020) 166501.

[3] K. J. J. Mayrhofer, J. C. Meier, S. J. Ashton, G. K. H.Wiberg, F. Kraus, M. Hanzlik, M. Arenz, Electrochem. Comm. 10 (2008) 1144-1147.

[4] F. Ruiz-Zepeda, M. Gatalo, A. Pavlišič, G. Dražić, P. Jovanovič, M. Bele, M. Gaberšček, N. Hodnik, Nano Lett. 19 (2019) 4919-4927.

[5] S. Rasouli, D. Myers, N. Kariuki, K. Higashida, N. Nakashima, P. Ferreira, Nano Lett. 19 (2019) 4653.

[6] N. de Jonge, L. Houben, R. E. Dunin-Borkowski, F. M. Ross, Nat. Rev. Mat. 4 (2019) 61-78.

[7] N. Hodnik, G, Dehm, K. J. J. Mayrhofer, Acc. Chem. Res. 49 (2016) 2015-2022. 